\title{
CAMERA: A system for fast and reliable acquisition of multiple ethological records
}

\author{
MAARTEN J. VAN DER VLUGT \\ iec ProGAMMA, Groningen, The Netherlands \\ MENNO R. KRUK and ANNEMOON M. M. VAN ERP \\ University of Leiden, Leiden, The Netherlands \\ and \\ REINT H. GEUZE \\ University of Groningen, Groningen, The Netherlands
}

\begin{abstract}
CAMERA is a system for collecting and editing ethological data. The hardware and software enable easy coding of complex behavioral interactions from video recordings. It is especially designed to improve accuracy, reliability, and training standards in coding behavior. The system also supports several basic analyses of collected data. CAMERA operates with both North American and European video signals.
\end{abstract}

The processing of directly observable live behavior, by the video recording and the coding of behavior into sequences of distinct events, is prone to error and distortion. Physiologists and physicists would be dismayed by an interrater reliability of less than $99.9 \%$, but students of behavior are often satisfied with a reliability of $85 \%$ or less. Moreover, subsequent analysis can only be as good as the coding allows. If the data are unreliable, the analysis may be meaningless.

There are many different errors possible during the coding of behavior. These errors originate from two sources: the observer and the coding instrument. How well the observer performs depends on the number of different events, the clarity of the definitions, the rate of occurrence of the events, the ergonomics of the technical task, and on vigilance and training. Also, improper feedback from the coding instrument to the observer while performing his or her task is an important factor of erratic coding.

This paper describes a system that has been developed to improve reliable and accurate coding of behavior. It is designed to reduce coding errors from both sources.

\section{Video Recording Using an Invisible Timecode}

Video recordings are often used in behavioral research. They make it possible to examine behavior repeatedly and, if necessary, at different speeds. Using video, one can code specific fast events at the appropriate slow speed, code different simultaneous behavioral traces, or code different individuals. Such codings can be merged into one

Correspondence should be addressed to $M$. J. van der Vlugt, iec ProGAMMA, P.O. Box 841,9700 AV Groningen, The Netherlands (e-mail:m.van.der.vlugt@gamma.rug.nl).

Note: One or more of the authors or their institutions have a financial interest in the hardware and software described in this paper-Editor single coding, provided that one has retained the same time frame for each coding.

CAMERA is a system designed to support the video coding task. The main components are shown in Figure 1. The system writes an invisible binary timecode redundantly in each video frame during recording, leaving the audio traces unaffected. During replay of the video recording at slow, normal, or high speed, the code is automatically retrieved and checked. Every time an event is coded on the event keyboard, the start and end time of the event, as well as a mnemonic label (a specific letter code, defined by the observer), is stored in the memory of a personal computer. The time corresponding to the current timecode is also directly visible on the video monitor. CAMERA is able to match timecodes in coded computer files and video frames. This allows the observer to search for certain episodes on tape, while inspecting or editing the corresponding coded files. Alternatively, one can search automatically for a specific part of a coded file, while viewing a videotape.

\section{A One-Key One-Event Keyboard}

CAMERA offers the observer a separate keyboard for coding. A specific event can be coded by pressing a corresponding button on the keyboard for the duration of the event. The keyboard also makes it possible to give the observer direct feedback on the coding process.

\section{Immediate Auditory and Visual Feedback}

Coding data from a visual record is very much like playing a piano in a noisy crowd, when the musician cannot hear the sounds produced. The player who gets no auditory feedback to guide his fingers may end up playing offkey. Ideally, during the coding of behavior, the coder's eyes should be on the video monitor instead of on the key- 


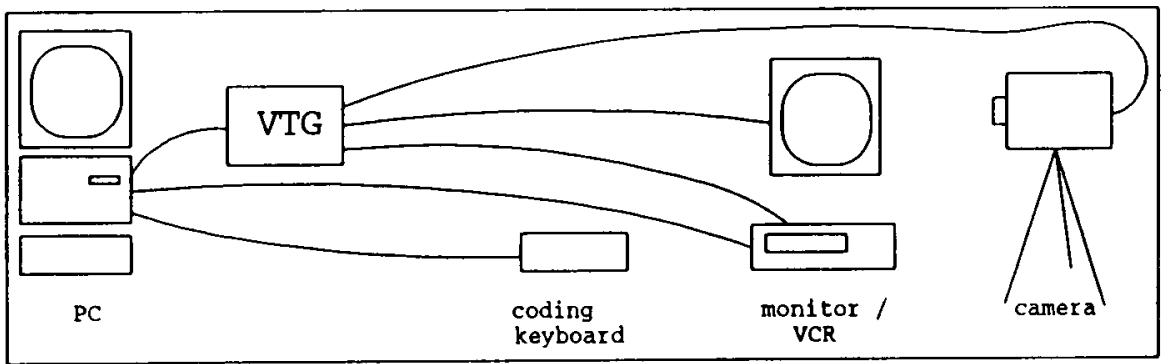

Figure 1. Main components of the CAMERA system. The system consists of a personal computer, video timecode generator (VTG), coding keyboard, video monitor, VCR, and video camera.

board or the personal computer monitor. However, that would force the observer to work like a pianist with blocked ears without any direct feedback. Moreover, while the musician may know in advance which note to play, the observer usually does not know the precise nature and timing of the next event, which is a major source of errors. Preventing errors while coding is essential, since the time needed to correct errors in a coded file increases with the number of errors.

Each button on the CAMERA keyboard generates a sound with a unique pitch when pressed, providing immediate auditory feedback. In addition, a specific mnemonic label (assigned by the observer to the specific event coded via that button) appears on the video monitor, providing immediate visual feedback. If several buttons are pushed simultaneously, the mnemonic labels appear side by side on the video screen. The label of the last button pushed appears at the right side of the label of the button pushed before it. Figure 2 shows an example of an image on the video monitor, together with mnemonic labels. We experienced that both immediate visual and immediate auditory feedback can reduce timing and recognition errors during coding.

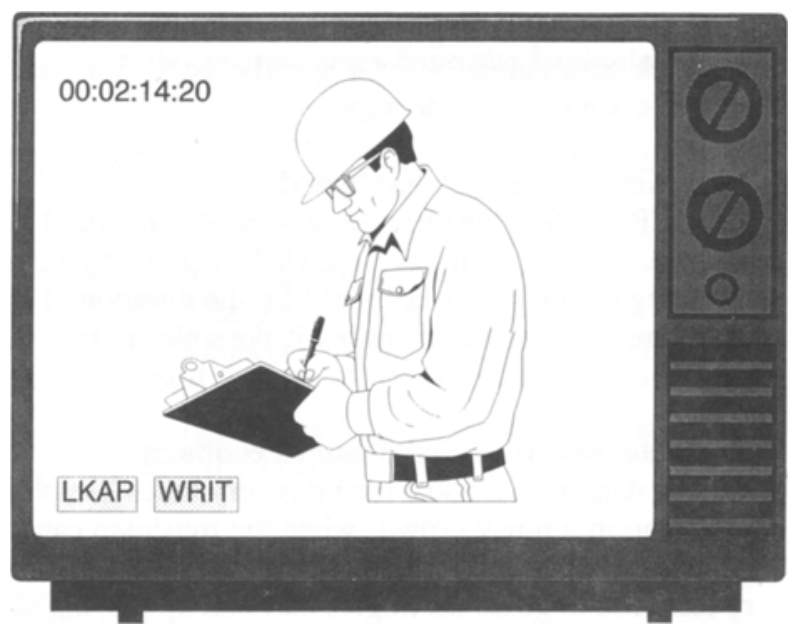

Figure 2. An example of an image on the video monitor while coding videotaped behavior, with time in the upper left corner and two mnemonic labels at the bottom. LKAP = "looking at paper"; WRTT = "writing."

\section{Visual Feedback During Playback}

Correcting errors in coded behavior files is timeconsuming and tedious. CAMERA uses the display of mnemonic labels on the video monitor to facilitate error correction. During playback of a coded file, CAMERA matches the timecode retrieved from the video frame with the timecode of the coded file. In playback, the mnemonic labels of the coded events also are displayed on the video monitor, in synchrony with the actual behavior. At the same time, the coded file scrolls on the personal computer monitor, and it may be edited. This allows convenient and fast detection and correction of coding errors. One also can use this facility to make a coding of a sequence of events for demonstrations, to train new observers, or to maintain one's own coding standards.

\section{Merging Independent Simultaneous Traces}

Even well-trained observers may find it difficult to code more than one individual at a time or to record positions or vocalizations simultaneous with actions. Such simultaneous, but independent, behavioral traces are best coded in separate runs and then merged as coded files. During replay, the mnemonic labels of the several traces can be displayed. This allows the observer to assess the interaction between different individuals or to study different aspects of behavior in one individual. The same facility allows the observer to code behavior first in general categories and subsequently in greater detail, without losing the time relation between the general and the specific behavioral trace. Analysis may force the observer to define a new event or to split an event into separate events. The merge facility makes it possible to code a newly defined event in a separate trace and merge it subsequently, without having to code the entire behavioral sequence again. As an example, Figure 3 shows an event plot of two aspects of the behavior "moving" coded in two runs.

\section{Training Observers and Improving Observer Reliability}

The visual feedback and merge facilities are especially useful when training new observers. An experienced observer can give a trainee a perfectly coded target file and a videotape of the behavior of interest. After viewing the replay of the target file and videotape several times, the 


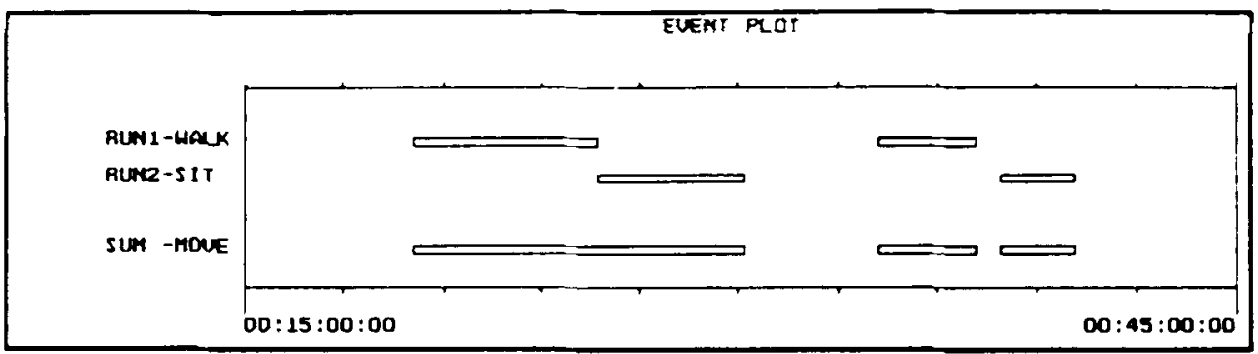

Figure 3. An event plot with events collected in two separate runs. In the first run (RUN1), "walking" (mnemonic WALK) is coded. In the second run (RUN2), "sitting" (mnemonic SIT) is coded. A new event "moving" (mnemonic MOVE) has been defined by combining "walking" and "stting." The new event is stored in a new trace (SUM).

trainee tries to match the observer's coding. Subsequently, the target file and the trainee's file are merged. Replaying the merged file in synchrony with the videotape of the behavior shows the trainee's coding and the correct coding, along with the actual behavior. Trainer and trainee can then observe differences in coding and discuss difficult cases. With this feature, new observers typically learn the coding scheme three to five times faster than they would without such training. The same procedure encourages two experienced observers to discuss definitions of events, evaluate the catalogue used to code behavior, and improve interobserver reliability.

\section{Inspecting the Results of Coding}

When the coded file has been checked carefully against the video behavior record, one may preview the results. While CAMERA has no advanced statistical functions, it provides elementary statistics on the frequency, average duration, and variation of the specific events coded. One can inspect the temporal structure of a sequence of multiple events with an event plot. One can also inspect the survivor functions of the durations of specific events, and the cumulative duration of specific events. These plots are a first step toward a more sophisticated anaiysis of behavior, such as Markov chain modeling. In CAMERA, these representations are used as powerful graphic tools for fast detection of temporal anomalies, change points, and transients in the behavioral record. The system also has facilities to export coded files in various ways for further analysis.

\section{Conclusion}

CAMERA contributes to reliable and accurate coding of behavior in various research environments. In addition, the system offers the possibility to inspect and verify already coded events. This feature appears to be valuable not only for judging the accuracy of the coding, but also for training new observers in a short time and without intensive guidance.

CAMERA, of course, does not solve all coding problems. For example, the circumstances under which a particular behavior can be considered to be present needs further investigation. Focal points are the identification of the features that characterize the presence or absence of a particular event, as well as the determination of threshold functions for the presence of a particular event (e.g., the minimum duration required for a feature to be recorded). Research on the criteria used by experienced observers in recognizing specific events might shed some light $\mathrm{cn}$ this problem. CAMERA has facilities that may support this type of research.

\section{Availability}

CAMERA is commercially available for PAL- and NTSC-type video systems. Contact iec ProGAMMA for more information (see $\mathbf{M}$. J. van der Vlugt's address). 